\title{
The association of foot structure and footwear fit with disability in children and adolescents with Down syndrome
}

\author{
Polly QX Lim', Nora Shields'², Nikolaos Nikolopoulos', Joanna T Barrett', Angela M Evans 1,3, Nicholas F Taylor ${ }^{2}$ \\ and Shannon E Munteanu $u^{1,3^{*}}$
}

\begin{abstract}
Background: Foot deformity, flat feet, and the use of ill-fitting footwear are common in children and adolescents with Down syndrome (DS). The aim of this study was to determine whether these observations are associated with foot-specific disability in this group.

Methods: A cross-sectional study design. Foot structure (foot posture determined using the Arch Index, presence of hallux valgus and lesser toe deformities) and footwear fit (determined by length and width percentage differences between the participant's foot and footwear) were assessed in 50 participants with DS (22 females, 28 males) aged five to 18 with a mean (SD) age of 10.6 (3.9) years. Foot-specific disability was determined using the parent-reported Oxford Ankle Foot Questionnaire for Children (OxAFQ-C). Associations between foot structure and footwear fit with the four domains (Physical, School and play, Emotional and Footwear) of the OXAFQ-C were determined using multivariate regression modelling.

Results: The mean (SD) Arch Index was 0.29 (0.08), and the prevalence of flat feet, hallux valgus and lesser toe deformities was $76 \%, 10 \%$ and $12 \%$ respectively. Few participants wore footwear that was too short (10\%), but the use of footwear that was too narrow was common (58\%). The presence of hallux valgus was significantly associated with increased disability for the OXAFQ-C School and play domain scores. The use of narrow-fitting footwear was significantly associated with increased levels of disability for the OxAFQ-C Physical, School and play, and Emotional domains. However, these variables only explained between $10 \%$ to $14 \%$ of the variance in the OXAFQ-C domain scores. There were no significant associations between foot structure and footwear fit with the OXAFQ-C Footwear domain scores.

Conclusions: Flatter feet and lesser toe deformities are not associated with foot-specific disability in children and adolescents with DS. Hallux valgus is associated with foot-specific disability during school and play activities. Ill-fitting footwear (too narrow) is common and is associated with foot-specific disability. Further research is required to identify if the relationship between narrow-fitting footwear and foot-specific disability is causal, and to identify other factors associated with foot-specific disability in children and adolescents with DS.
\end{abstract}

Keywords: Foot, Shoes, Flat foot, Foot deformities, Hallux valgus, Down syndrome

\footnotetext{
* Correspondence: s.munteanu@latrobe.edu.au

'Discipline of Podiatry, College of Science, Health and Engineering, La Trobe

University, Bundoora, Victoria 3086, Australia

${ }^{3}$ Lower Extremity and Gait Studies Program, La Trobe University, Bundoora,

Victoria 3086, Australia

Full list of author information is available at the end of the article
} 


\section{Background}

Down syndrome (DS), also known as Trisomy 21, is the most prevalent chromosomal disorder and is caused by the presence of all or part of a third copy of chromosome $21[1,2]$. DS is primarily characterised by variable intellectual disability, distinct facial phenotype, short stature, generalised joint laxity and hypotonia [2,3]. Physical disability is an additional problem in people with DS and manifests as reduced physical fitness [4], reduced lower limb muscle strength [5], less functional gait patterns [6] and gait instability [7].

Musculoskeletal foot disorders are prevalent in individuals with DS [8-15]. In a population-based study of 197 young adults with DS aged 15 to 30 years, foot problems were reported to be the most prevalent muscle and bone condition, affecting nearly two-thirds of participants [12]. Of these participants, $66 \%$ reported that foot problems frequently affected their daily life [12]. Other studies have shown that children and adolescents with DS are also more likely to have foot conditions than their peers without DS [16-18]. There is evidence that children and adolescents with DS have flatter feet [16-18] and are more likely to have structural foot disorders such as hallux valgus [16]. Due to the presence of hallux valgus, footwearfitting problems have been a concern in individuals with DS [11,17]. There is evidence to suggest that children with DS were more likely to wear ill-fitting footwear [17].

While it has been recognised that children with DS are more likely to have flatter feet, hallux valgus and footwear-fitting problems, no study has attempted to determine if there is a relationship between these characteristics with foot-specific disability in this group. Therefore, the aim of this study was to determine if there is an association between foot structure and footwear fit with the extent of disability related to foot problems in children and adolescents with DS.

\section{Methods}

This cross-sectional study was approved by the Human Ethics Committee of La Trobe University, Australia (HEC13-035).

\section{Recruitment}

Children and adolescents with all genetic variants of DS aged five to 18 years were invited to participate in this study through email advertisements and flyers distributed to members of Down Syndrome Victoria. Participants were excluded if they had any previous lower limb surgery, were unable to walk without a supportive device such as a walker or brace, or had a concomitant medical condition or injury that could affect their physical function (e.g. neurological or inflammatory disorder). Fifty children and adolescents with DS aged five to 18 years were recruited. The participant recruitment process is shown in Figure 1.

\section{Data collection}

From October 2013 to May 2014, assessments were conducted at a university health sciences clinic (La Trobe University, Melbourne, Victoria, Australia) by the same investigator (PQXL). Written informed consent was obtained from all parents or guardians prior to data collection. Where possible, consent was also obtained from participants aged 13 years and above. All of the participants' parent or guardian completed a participant information questionnaire concerning the participants' medical history and current medications prior to the assessment. The participants' age, sex, height $(\mathrm{cm})$, weight $(\mathrm{kg})$, body mass index $\left(\mathrm{kg} / \mathrm{m}^{2}\right)$, and use of foot orthoses were documented.

\section{Assessment of foot structure Foot posture}

Foot posture was assessed using the Arch Index [19]. The Arch Index was calculated by obtaining a footprint using PressureStat ${ }^{\mathrm{TM}}$ carbon paper (Bailey Instruments Ltd, UK) with the participant in a relaxed weight-bearing stance position. The weight-bearing length of the footprint $(\mathrm{cm})$ was determined by measuring the length from the centre of the base of the heel to the centre of the front edge of the forefoot (excluding the toes). The front edge was determined as the front weight-bearing border of the forefoot marked on a line extending from the second digit in the transverse plane [20]. Areas of forefoot, midfoot, and rearfoot regions were then determined by dividing the length of the foot into equal thirds [20]. The surface area of each third of the weight-bearing regions was calculated using a graphics tablet and stylus (Wacom Technology, Vancouver, Canada) and an image analysis software Scion ${ }^{\circ}$ (Scion Corporation, Chicago, Illinois, USA). The Arch Index was then determined as the ratio of area of middle third of the footprint to the entire footprint excluding the toes, with a higher ratio indicating a flatter foot [19]. This measure has been shown to exhibit excellent reproducibility in child and adolescent populations [21,22] and has been validated against radiographs of the foot [23]. There is evidence that assessment of the Arch Index can be affected by body composition [24,25]; this may be a concern as people with DS are more likely to be overweight $[2,26,27]$. However, recent work has shown that measures of Arch Index are not affected by body mass index in children and adolescents with DS [28]. 


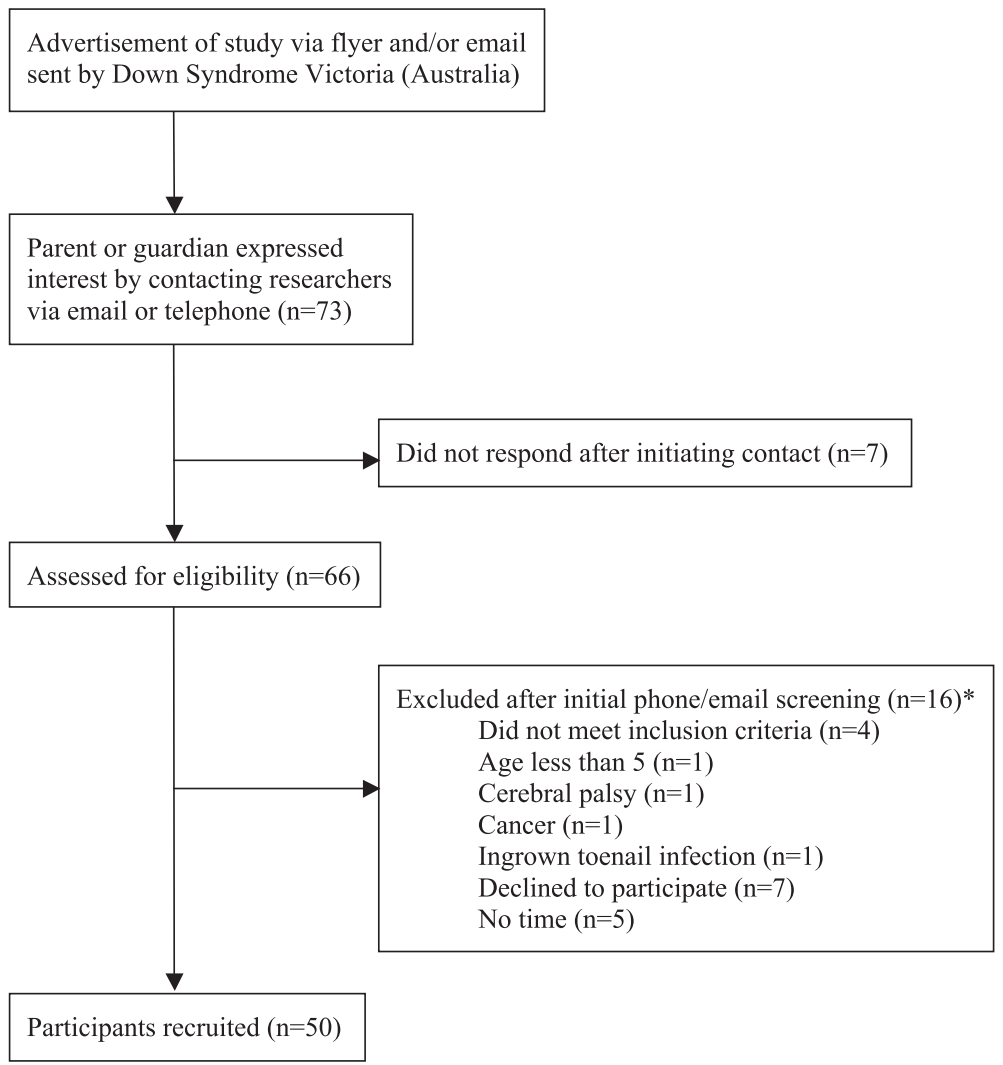

Figure 1 Participant recruitment flowchart. *Some volunteers were excluded for multiple reasons.

\section{Presence of hallux valgus and lesser toe deformities}

The severity of hallux valgus was determined using the Manchester scale grading system [29]. This measure has good reproducibility (kappa coefficients $=0.80$ to 0.89 ) $[29,30]$ and has been validated against radiographic measurements [31]. The degree of deformity was graded on a scale of 0 to 3 (no deformity to mild, moderate and severe deformity) based on observation of the participant's foot in a relaxed bipedal stance. Scores for Manchester scale of hallux valgus were dichotomised where a score of 0 or 1 was categorised as absent, and scores of 2 and 3 were categorised as present for hallux valgus [31]. Manchester scale grades of 2 (moderate deformity) and 3 (severe deformity) were chosen to represent the presence of hallux valgus as previous work by Garrow et al. [29] has shown that raters have little difficulty distinguishing between mild (score 1) and moderate (score 2) deformity. However, raters have difficulty distinguishing between no deformity (score 0) and mild deformity (score 1). In addition, previous work, using radiographic assessment as the gold standard, has shown that although there are significant differences in the mean hallux abductus angle between all Manchester scale grades, the total range of values overlaps for the 'none' and 'mild' deformity grades, indicating that differentiation between milder levels of deformity may not always be accurate [31]. Lesser toe deformities were documented by observing the presence of sagittal plane deformities (that is, claw, hammer or mallet toes) of the second to fifth digits while the participant was standing in a relaxed weight-bearing position. This assessment has good reproducibility as inter-tester reliability has previously been shown to have an absolute agreement of $85 \%$ [32].

\section{Footwear fit assessment}

Footwear fit of the participants' most regularly worn footwear was measured using the technique described previously [33]. The outline of the sole of each shoe was traced onto graph paper. A tracing of participant's foot was performed while the participant stood in a relaxed bipedal position on the PressureStat ${ }^{\mathrm{TM}}$ footprint mat. Footwear and foot tracings were then retraced using a computer graphics tablet and stylus (Wacom Technology, Vancouver, Canada) in conjunction with an image analysis software Scion ${ }^{\bullet}$ (Scion Corporation, Chicago, Illinois, USA). In cases where the upper of the footwear was wider than the sole, the boundary of the upper was used in the analysis [33]. The maximum length and width of the participant's foot and footwear were documented in millimetres. This method has sound intra-rater reproducibility 
(ICCs $>0.99$, coefficients of variation between 0.3 and 1.2\%) [33]. The percentage difference between the foot and footwear dimensions was calculated for length and width measurements. Positive values indicate that the footwear dimension is larger than the corresponding foot dimension.

\section{Assessment of foot-specific disability}

The disability associated with foot and ankle problems was assessed using the Oxford Ankle Foot Questionnaire for Children (OxAFQ-C) [34]. The 15-item questionnaire assesses the severity of foot and ankle problems across three domains of disability, (i) Physical (6 items), (ii) School and play (4 items), and (iii) Emotional (4 items). A final domain (item 15: Footwear) is not considered to be an assessment of disability [34], however it was included in our analysis as it is considered to reflect the concern of whether the participant's foot or ankle stopped them from wearing the footwear they wanted to wear [34]. The scoring system assesses how frequently each issue (represented by an item in the questionnaire) is a problem. The response options to each item are on a 5-point scale rated from never (4), rarely (3), sometimes (2), very often (1) to always (0). Domain scores were calculated as the total of the scale item scores divided by the maximum for each domain (i.e. Physical 24, School and play 16, Emotional 16 and Footwear 4). Domain scores were then transformed to a percentage scale (0 to 100\%) [34]. A lower score for a domain represents greater disability [34].

The OxAFQ-C is available in a child- or parent (proxy)-reported version. We administered the parent version of the OxAFQ-C because it was assumed that the child version would not be reflective of the participant's response as the participants would be unable to provide responses given their level of intellectual disability and age (e.g. 5 years old). The OxAFQ-C has demonstrated good child-parent agreement for all OxAFQ-C domains (Physical ICC $=0.78$; School and play ICC = 0.84; Emotional $\mathrm{ICC}=0.73$; and Footwear $\mathrm{ICC}=0.65$ ). The OxAFQ-C has also demonstrated both crosssectional and longitudinal validity $[34,35]$ as an assessment tool across a range of paediatric conditions of the foot and ankle [36].

\section{Sample size determination}

Using G*power 3.1 software [37] for performing a sample size calculation, we estimated that we would need a minimum of 46 participants to detect a significant association between the foot structure and footwear fitting variables with each domain of the OxAFQ-C using linear regression models. This sample size was estimated using a $p$ value $=0.05$, power $=0.8$, effect size $\left(R^{2}\right)=0.15$ for each model, and number of predictors $=4$ (each foot structure and footwear fitting variable, with age, sex, body mass index).

\section{Statistical analysis}

All analyses were performed using IBM SPSS Statistics for Windows, Version 22.0 (Armonk, NY: IBM Corp.) and $p$ values $<0.05$ were considered statistically significant. Data were assessed for normality and any non-normally distributed data were transformed using natural logarithm (Ln) where possible. In order to satisfy the independence assumption of statistical analysis, only measurements from the participant's right foot and footwear were analysed [38].

We initially explored our data by analysing univariate associations (assumes that the response variable is influenced only by a single factor) between potentially associated variables with the domain scores of the OxAFQ-C using correlation statistics, independent samples $t$-tests, or Mann-Whitney U-tests (specific approach depended on the scaling and distribution of the data).

To determine the relationship between foot structure and footwear fit variables with the domains of the OxAFQ-C we used a hierarchical multivariate regression approach to determine the relationship after adjusting for age, sex and body mass index. This statistical approach allowed us to determine the relationship between potentially associated variables with the domain scores of the OxAFQ-C when controlling for confounding factors (i.e. age, sex and body mass index). In these analyses, footwear fit was dichotomised into two categories by transforming the percentage difference (between dimensions of the shoe and foot) into medians. The lower median represented poorer fitting footwear (i.e. footwear of insufficient length and width) and the upper median represented better footwear fit (i.e. footwear of appropriate length and width).

We used multivariate linear regression models for analyses where the Physical and School and play domains of the OxAFQ-C were the outcome variables. The fraction of variance independently explained by the foot structure or footwear fit variables was determined using the $R^{2}$ change statistic and the associated $p$ value. We used multivariate logistic regression models for the analyses where the Emotional and Footwear domains of the OxAFQ-C were the outcome variables as we were unable to transform these variables to be normally distributed. For each variable in the logistic regression models, odds ratios (OR) and 95\% confidence intervals (CI) were determined (after adjusting for sex, age and body mass index). The fraction of variance independently explained by the foot structure or footwear fit variables was determined using the change in Nagelkerke $R^{2}$ statistic and the associated $p$ value [39]. 


\section{Results}

Participant characteristics are shown in Table 1. Twentyeight (56\%) of participants were male, and the group had a mean (SD) age of 10.6 (3.9) years (range five to 18 years). Thirty-eight participants (76\%) were classified as having flat feet, five (10\%) had hallux valgus (all were classified as moderate severity) and six (12\%) had lesser toe deformities. Five participants (10\%) wore footwear

Table 1 Participant characteristics

\begin{tabular}{lll}
\hline Characteristic & Value & Range \\
\hline General & & \\
Age (years), mean (SD) & & \\
Sex, n (\%) male & $10.6(3.9)$ & $\mathrm{N} / \mathrm{A}$ \\
Type of DS, n (\%) & $28(56)$ & $\mathrm{N} / \mathrm{A}$ \\
Trisomy 21/Translocation/Mosaic & $44(88) / 5(10) / 1(2)$ \\
Any type of medication, n (\%) & $23(46)$ & $\mathrm{N} / \mathrm{A}$ \\
Height (cm), mean (SD) & $131.9(18.6)$ & 96.0 to \\
& & 164.5 \\
Weight (kg), mean (SD) & $39.6(18.4)$ & 16.3 to 85.1 \\
Body mass index (kg/m²), mean & & \\
(SD) & & 13.8 to 34.9 \\
Overall & $21.5(5.3)$ & 14.7 to 33.8 \\
Males & $21.1(4.9)$ & 13.8 to 34.9 \\
Females & $22.1(5.8)$ &
\end{tabular}

Foot structure

$\begin{array}{lll}\text { Arch Index, mean (SD) } & 0.29(0.08) & 0.01 \text { to } 0.39 \\ \begin{array}{l}\text { Foot posture, } \mathrm{n}(\%)^{\mathrm{b}} \\ \text { Flat/typical/high arch }\end{array} & \begin{array}{l}38(76) / 6(12) / 6 \\ (12)\end{array} & \mathrm{N} / \mathrm{A} \\ \text { Hallux valgus, } \mathrm{n}(\%) & 5(10) & \mathrm{N} / \mathrm{A} \\ \text { Lesser toe deformity, n (\%) } & 6(12) & \\ \text { Footwear fit } & & -14.3 \text { to } \\ \text { Length (\%) } & 9.0(7.5) & 23.3 \\ & & -26.5 \text { to } \\ \text { Width (\%) } & -4.5(10.0) & 13.6\end{array}$

OxAFQ-C domain scores, mean (SD)

$\begin{array}{lll}\text { Physical }^{d}(\%) & 72.8(21.4) & 33.3 \text { to } \\ & & 100.0 \\ & 84.9(18.1) & 31.3 \text { to } \\ & & 100.0 \\ \text { School and play } & \text { d (\%) } & 43.8 \text { to } \\ & 92.9(13.8) & 100.0 \\ \text { Emotional }^{d}(\%) & & 0.0 \text { to } 100.0 \\ & 67.5(3.9) & \end{array}$

${ }^{\mathrm{a} S D}$ : Standard deviation.

${ }^{b}$ Based on the Arch Index, foot postures were classified as follows: Arch Index $\geq 0.26$ (flat), $0.22<$ Arch Index $<0.26$ (typical), Arch Index $\leq 0.21$ (high arch) [19].

'Percentage difference between shoe and foot dimensions. Positive values indicate shoe dimension greater than foot dimension. Measurements were based on $n=48$ due to missing data.

dParent-reported Oxford Ankle Foot Questionnaire for Children (OxAFQ-C) domain scores were based on $n=49$ due to missing data. shorter than their feet, and 29 (58\%) wore footwear narrower than their feet. The mean (SD) of the OxAFQ-C Physical domain score was $72.8 \%$ (21.4), the OxAFQ-C School and play domain score was $84.9 \%$ (18.1), the OxAFQ-C Emotional domain score was 92.9\% (13.8), and the OxAFQ-C Footwear domain score was $67.5 \%$ (3.9) (Table 1).

\section{Univariate associations between participant characteristics,} foot structure and footwear fit with OXAFQ-C domain scores Pearson's product moment correlations $(r)$ and Spearman's rank-order correlations $(\rho)$ for the associations between participant characteristics (continuous scaled variables) and OxAFQ-C domain and item scores are shown in Table 2. The age, height and weight of participants were significantly inversely associated with the OxAFQ-C Emotional domain scores $(\rho=-0.37, p=0.009 ; \rho=-0.34$, $p=0.016 ; \rho=-0.34, p=0.018$ respectively). Body mass index was significantly inversely associated with the OxAFQ-C School and play domain scores $(r=-0.30$; $p=0.039$ ).

No associations were found between Arch Index and any of the OxAFQ-C domain scores (Table 2). Mean differences and 95\% confidence intervals (CI) for the associations between dichotomous scaled independent variables and the OxAFQ-C Physical, School and play, Emotional, and Footwear domain scores are shown in Table 3. No statistically significant differences were found between sexes with any OxAFQ-C domain scores. Participants with hallux valgus had significantly reduced OxAFQC School and play domain scores (mean difference $-19.3 \%$, 95\% CI -37.7 to $-1.0, p=0.040$ ). Participants with lesser toe deformities had significantly reduced OxAFQ-C Emotional domain scores (mean difference $-11.5 \%$, $95 \%$ CI -29.0 to $6.0, p=0.047$ ) (Table 3).

Measures of the difference between the length of the participant's foot and footwear were not significantly associated with any OxAFQ-C domain scores. However, the percentage difference between the width of the participant's foot and footwear was significantly positively associated with three OxAFQ-C domain scores (Physical: $r=0.34, p=0.019$; School and play: $r=0.44, p=$ 0.002, Emotional: $\rho=0.35, p=0.014$ respectively). These results show that increasing severity of ill-fitting footwear (narrowness) is associated with lower OxAFQ-C Physical, School and play and Emotional domain scores. There were no significant associations between footwear fit (length or width) with the OxAFQ-C Footwear domain scores (Table 2).

\section{Multivariate associations between foot structure and footwear fit with OxAFQ-C domain scores}

Multivariate associations between foot structure and the fit of footwear with the OxAFQ-C domain scores are 
Table 2 Univariate associations (correlations) between continuous scaled variables and OxAFQ-C domain scores

\begin{tabular}{|c|c|c|c|c|c|c|c|c|}
\hline \multirow[t]{2}{*}{ Participant characteristics } & \multicolumn{2}{|l|}{ Physical $^{a}$} & \multicolumn{2}{|c|}{ School and play ${ }^{a}$} & \multicolumn{2}{|l|}{ Emotional $^{\mathrm{a}}$} & \multicolumn{2}{|l|}{ Footwear } \\
\hline & $\begin{array}{l}\text { Univariate } \\
\text { association }^{\mathbf{b}}\end{array}$ & $p$ value & $\begin{array}{l}\text { Univariate } \\
\text { association }^{\mathbf{b}}\end{array}$ & $p$ value & $\begin{array}{l}\text { Univariate } \\
\text { association }^{c}\end{array}$ & $p$ value & $\begin{array}{l}\text { Univariate } \\
\text { association }\end{array}$ & $p$ value \\
\hline Age (years) & -0.155 & 0.289 & -0.190 & 0.191 & -0.370 & 0.009 & 0.020 & 0.889 \\
\hline Height $(\mathrm{cm})$ & -0.192 & 0.186 & -0.198 & 0.172 & -0.342 & 0.016 & -0.009 & 0.949 \\
\hline Weight (kg) & -0.274 & 0.056 & -0.273 & 0.058 & -0.338 & 0.018 & -0.070 & 0.630 \\
\hline Body mass index $\left(\mathrm{kg} / \mathrm{m}^{2}\right)$ & -0.278 & 0.053 & -0.295 & 0.039 & -0.272 & 0.059 & -0.199 & 0.166 \\
\hline Arch Index & -0.236 & 0.102 & -0.245 & 0.090 & -0.127 & 0.385 & -0.148 & 0.306 \\
\hline $\begin{array}{l}\text { Percentage difference between length of foot } \\
\text { and footwear }{ }^{d}(\%)\end{array}$ & 0.069 & 0.642 & 0.048 & 0.745 & 0.157 & 0.286 & -0.058 & 0.694 \\
\hline $\begin{array}{l}\text { Percentage difference between width of foot } \\
\text { and footwear }{ }^{d}(\%)\end{array}$ & 0.338 & 0.019 & 0.437 & 0.002 & $0.353^{c}$ & 0.014 & 0.064 & 0.664 \\
\hline
\end{tabular}

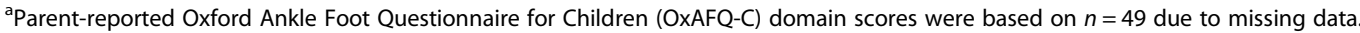

bearson's product moment correlation $(r)$.

'Spearman's rank-order correlation $(\rho)$.

${ }^{d}$ Correlation analyses were based on $n=48$ due to missing data.

shown in Tables 4, 5, 6 and 7. The values that defined the lower median for the variable ill-fitting footwear were as follows: length (shoe $14.3 \%$ shorter to $10.8 \%$ longer than foot), width (shoe $26.5 \%$ to $4.9 \%$ smaller than foot).

\section{OXAFQ-C Physical domain}

There was no significant association between foot structure and the OxAFQ-C Physical domain scores (Table 4). The use of footwear with an inappropriate length was not associated with the OxAFQ-C Physical domain scores. However, the use of footwear that was an inappropriate width was significantly associated with the OxAFQ-C Physical domain scores (in adjusted analyses, $R^{2}$ change $\left.=0.099, p=0.027\right)$ (Table 4$)$.

\section{OXAFQ-C School and play domain}

Arch Index, lesser toe deformities and the use of footwear with an inappropriate length were not associated with the OxAFQ-C School and play domain scores (Table 5). The presence of hallux valgus was significantly associated with the OxAFQ-C School and play domain scores (in adjusted analyses, $R^{2}$ change $=0.080, p=0.046$ ). The use of footwear that was an inappropriate width was significantly associated with the OxAFQ-C School and play domain scores (in adjusted analyses, $R^{2}$ change = $0.137, p=0.009)$ (Table 5).

\section{OXAFQ-C Emotional domain}

The use of footwear that was an inappropriate width was significantly associated with the OxAFQ-C Emotional domain scores (in adjusted analyses OR 5.11, 95\% CI 1.26 to 20.70, change in Nagelkerke $R^{2}=0.135$, $p=0.022$ ) (Table 6). In adjusted analyses, age, sex, body mass index and the presence of lesser toe deformities were not significantly associated with the OxAFQ-C Emotional domain scores (Table 6).

\section{OXAFQ-C Footwear domain}

None of the foot or footwear fit characteristics were significantly associated with the OxAFQ-C Footwear domain scores (Table 7).

Table 3 Univariate associations (differences between groups) between dichotomous scaled variables and OxAFQ-C domain scores

\begin{tabular}{|c|c|c|c|c|c|c|c|c|}
\hline \multirow{2}{*}{$\begin{array}{l}\text { Participant } \\
\text { characteristics }\end{array}$} & \multicolumn{2}{|l|}{ Physical $^{\mathrm{a}, \mathrm{b}}$} & \multicolumn{2}{|l|}{ School and play ${ }^{a}$} & \multicolumn{2}{|l|}{ Emotional $^{\mathrm{a}}$} & \multicolumn{2}{|l|}{ Footwear } \\
\hline & $\begin{array}{l}\text { Mean difference } \\
(95 \% \mathrm{Cl})\end{array}$ & $p$ value & $\begin{array}{l}\text { Mean difference } \\
(95 \% \mathrm{Cl})\end{array}$ & $p$ value & $\begin{array}{l}\text { Mean difference } \\
(95 \% \mathrm{Cl})\end{array}$ & $p$ value $^{c}$ & $\begin{array}{l}\text { Mean difference } \\
(95 \% \mathrm{CI})\end{array}$ & $p$ value $^{c}$ \\
\hline $\operatorname{Sex}^{d}$ & $-2.0(-14.4$ to 10.5$)$ & 0.754 & $-1.5(-12.1$ to 9.0$)$ & 0.771 & $-5.2(-13.1$ to 2.7$)$ & 0.350 & $6.9(-8.8$ to 22.6$)$ & 0.299 \\
\hline Hallux valgus ${ }^{e}$ & $-19.1(-41.1$ to 2.8$)$ & 0.062 & $-19.3(-37.7$ to -1.0$)$ & 0.040 & $-10.9(-37.8$ to 15.9$)$ & 0.133 & $-25.0(-50.1$ to 0.1$)$ & 0.123 \\
\hline $\begin{array}{l}\text { Lesser toe } \\
\text { deformity }^{e}\end{array}$ & $-10.9(-25.9$ to 16.0$)$ & 0.297 & $-13.9(-30.8$ to 3.0$)$ & 0.106 & $-11.5(-29.0$ to 6.0$)$ & 0.047 & $-19.9(-43.3$ to 3.6$)$ & 0.199 \\
\hline
\end{tabular}


Table 4 Multivariate linear regression analyses with OXAFQ-C Physical domain scores as the dependent variable $^{a, b}$

\begin{tabular}{|c|c|c|}
\hline \multirow[b]{2}{*}{ Independent variables } & \multicolumn{2}{|c|}{ OxAFQ-C Physical domain score ${ }^{c}$} \\
\hline & $R^{2}$ change & $p$ value for $R^{2}$ change \\
\hline \multicolumn{3}{|l|}{ Foot structure } \\
\hline Arch Index & 0.032 & 0.212 \\
\hline Hallux valgus (presence) & 0.066 & 0.070 \\
\hline Lesser toe deformity (presence) & 0.014 & 0.409 \\
\hline \multicolumn{3}{|l|}{ Footwear fit ${ }^{d}$} \\
\hline Length (ill-fitting/lower median) ${ }^{e}$ & 0.013 & 0.431 \\
\hline Width (ill-fitting/lower median) ${ }^{\mathrm{e}}$ & 0.099 & 0.027 \\
\hline
\end{tabular}

${ }^{a}$ Adjusted for sex, age and body mass index.

${ }^{\mathrm{b}}$ Parent-reported Oxford Ankle Foot Questionnaire for Children (OxAFQ-C) domain scores were based on $n=49$ due to missing data.

'Analyses performed on transformed OxAFQ-C Physical domain scores.

${ }^{d}$ Linear regression analyses performed for footwear fit were based on $n=48$ due to missing data.

eIII-fitting: representing the greatest disparity between the participant's foot and footwear length and width dimensions; expressed as a percentage. The values that defined the lower median (ill-fitting) were as follows: length (shoe $14.3 \%$ shorter to $10.8 \%$ longer than foot), width (shoe $26.5 \%$ to $4.9 \%$ narrower than foot).

\section{Discussion}

Foot problems are the most frequently reported musculoskeletal disorders in children and adolescents with DS [12]. Foot-related characteristics such as flat feet, hallux valgus and ill-fitting footwear are common. However, until now, no studies have specifically investigated the association between foot structure and footwear fit with foot-specific disability in children and adolescents with DS. We found that the presence of hallux valgus was significantly associated with foot-specific disability during

\begin{tabular}{|c|c|c|}
\hline \multirow[b]{2}{*}{ Independent variables } & \multicolumn{2}{|c|}{$\begin{array}{l}\text { OxAFQ-C School and play domain } \\
\text { score }\end{array}$} \\
\hline & $R^{2}$ change & $p$ value for $R^{2}$ change \\
\hline \multicolumn{3}{|l|}{ Foot structure } \\
\hline Arch Index & 0.038 & 0.174 \\
\hline Hallux valgus (presence) & 0.080 & 0.046 \\
\hline Lesser toe deformity (presence) & 0.037 & 0.179 \\
\hline \multicolumn{3}{|l|}{ Footwear fit ${ }^{c}$} \\
\hline Length (ill-fitting/lower median) ${ }^{d}$ & 0.030 & 0.232 \\
\hline Width (ill-fitting/lower median) ${ }^{d}$ & 0.137 & 0.009 \\
\hline
\end{tabular}

${ }^{a}$ Adjusted for sex, age and body mass index.

barent-reported Oxford Ankle Foot Questionnaire for Children (OXAFQ-C) domain scores were based on $n=49$ due to missing data.

'Linear regression analyses performed for footwear fit were based on $n=48$ due to missing data.

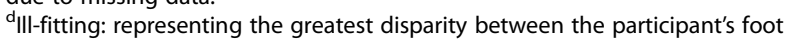
and footwear length and width dimensions; expressed as a percentage.

The values that defined the lower median (ill-fitting) were as follows: length (shoe $14.3 \%$ shorter to $10.8 \%$ longer than foot), width (shoe $26.5 \%$ to $4.9 \%$ narrower than foot). school and play activities. However, foot posture (that is, the severity of flat footedness) and lesser toe deformities were not associated with foot-specific disability. In addition, we found that inappropriately fitting footwear was associated with foot-specific disability. The OxAFQ-C Physical, School and play, and Emotional domain scores were significantly associated with the use of footwear that was too narrow. In contrast, there were no associations between the length fit of footwear with foot-specific disability. There was also no association found between foot structure and footwear fit with the OxAFQ-C Footwear domain scores. This finding suggests parents' perceptions of their child's ability to wear their shoe of choice is not associated with their child's foot structure or fit of the footwear.

Flat feet were prevalent in our participants, which is similar to previous studies $[6,16-18,28,40,41]$. We hypothesised that a flat foot posture would be associated with footspecific disability based on previous studies showing that children with DS and flat feet have reduced gait efficiency [6,41] and impaired foot function [16]. However, our findings showed that foot posture was not associated with footspecific disability. One explanation could be that although flat footedness is associated with changes in biomechanics $[6,16,41]$, there are no adverse effects on their ability to walk, run or engage in school and play activities (as measured using the OxAFQ-C Physical and School and play domain scores). This is supported by the notion that a paediatric flat foot is not recognised as a medical condition in the general population and children can present with a flat foot that is largely asymptomatic $[42,43]$.

In this study, hallux valgus was associated with footspecific disability during school and play activities. This is not surprising given that hallux valgus has been associated with greater levels of foot-specific disability in other populations $[44,45]$. We did not find any associations between lesser toe deformity and foot-specific disability, in contrast to older populations where deformity is associated with impaired balance and functional capacity [32]. A possible reason could be that our participants were younger and may not have been exposed to digital deformities for long enough for any adverse consequences (such as the formation of corns and calluses) to develop [33].

We found that the use of ill-fitting footwear was associated with increased levels of foot-specific disability. Specifically, there was an association between the width of footwear relative to foot width (shoes too narrow) and increased levels of OxAFQ-C disability scores, suggesting the use of footwear that is too narrow is associated with increased levels of foot-specific disability. Our findings are broadly in agreement with studies of older populations showing increased foot pain (disability) with the wearing of ill-fitting footwear $[33,46]$. In contrast, there was no association with length fit of the footwear. Our 
Table 6 Multivariate logistic regression analyses with OxAFQ-C Emotional domain scores as the dependent variable ${ }^{a, b}$

\begin{tabular}{|c|c|c|c|}
\hline \multirow[b]{2}{*}{ Independent variables } & \multicolumn{3}{|c|}{ OxAFQ-C Emotional domain score ${ }^{c}$} \\
\hline & OR $(95 \% \mathrm{Cl})^{\mathrm{d}}$ & Change in Nagelkerke $R^{2}$ & $p$ value \\
\hline \multicolumn{4}{|l|}{ Foot structure } \\
\hline Arch Index ${ }^{e}$ & 1.06 (0.97 to 1.16$)$ & 0.174 & 0.217 \\
\hline Hallux valgus (presence) & $10.48(0.76$ to 144.17$)$ & 0.112 & 0.121 \\
\hline Lesser toe deformity (presence) & 3.92 (0.33 to 46.75$)$ & 0.128 & 0.279 \\
\hline \multicolumn{4}{|l|}{ Footwear fit $^{f}$} \\
\hline Length (ill-fitting/lower median) ${ }^{9}$ & 3.78 (0.88 to 16.16$)$ & 0.199 & 0.073 \\
\hline Width (ill-fitting/lower median) $)^{9}$ & 5.11 (1.26 to 20.70$)$ & 0.135 & 0.022 \\
\hline
\end{tabular}

${ }^{\mathrm{a}}$ Adjusted for sex, age and body mass index.

bParent-reported Oxford Ankle Foot Questionnaire for Children (OxAFQ-C) domain scores were based on $n=49$ due to missing data.

'OxAFQ-C Emotional domain scores were dichotomised where the lowest quartile was classified as 'disability' and the upper three quartiles were classified as 'no disability'.

${ }^{\mathrm{d}}$ OR: Odds ratio.

${ }^{\mathrm{e}}$ Arch Index multiplied by hundred.

fLogistic regression analyses performed for footwear fit were based on $n=48$ due to missing data.

III-fitting: representing the greatest disparity between the participant's foot and footwear length and width dimensions; expressed as a percentage. The values that defined the lower median (ill-fitting) were as follows: length (shoe $14.3 \%$ shorter to $10.8 \%$ longer than foot), width (shoe $26.5 \%$ to $4.9 \%$ narrower than foot).

strategy to dichotomise footwear into appropriate or illfitting categories using median values as a threshold, resulted in an inappropriate shoe length as being $14.3 \%$ shorter to $10.8 \%$ longer than foot. It is widely accepted that footwear needs to be longer than the foot to allow elongation when weight-bearing [47], so it could be argued that our cut-off may have masked any associations if they existed. However, we analysed the association between footwear length and OxAFQ-C scores using a cut-off where footwear was classified as being of inadequate length was shorter than the actual length of the foot. Again, we did not find any significant associations between footwear that was shorter than the foot with foot-specific disability. Therefore, we believe our findings are robust. An explanation as to why there was a relationship between footwear width (and not length) with foot-specific disability could pertain to the 'typical' foot shape of children with DS. In particular, children with DS have a wider forefoot (related to the presence of hallux valgus, a greater first intermetatarsal angle or a flat foot posture) compared to children without DS [16]. Hence, children with DS are more likely to wear shoes of an inadequate width as a result of their wider forefoot.

There are limitations to the current study. First, we were limited to using the parent version of the OxAFQ$\mathrm{C}$ because of anticipated difficulties in getting children and adolescents with DS to independently complete the questionnaire. Although previous work has shown that the OxAFQ-C parent-reported domain scores show moderate agreement with the child-reported domain scores, the agreement is not perfect [34]. In addition, although the OxAFQ-C has been shown to demonstrate satisfactory construct validity overall, the validity does vary across domains and is also influenced by parent or child completion. For example, the validity of the OxAFQ-C School and play domain is greater using child- than parent-

Table 7 Multivariate logistic regression analyses with OxAFQ-C Footwear domain scores as the dependent variable ${ }^{a}$

\begin{tabular}{|c|c|c|c|}
\hline \multirow[b]{2}{*}{ Independent variables } & \multicolumn{3}{|c|}{ OxAFQ-C Footwear ${ }^{b}$ domain score } \\
\hline & OR $(95 \% \mathrm{Cl})^{c}$ & Change in Nagelkerke $R^{2}$ & $p$ value \\
\hline \multicolumn{4}{|l|}{ Foot structure } \\
\hline Arch Index ${ }^{d}$ & 1.03 (0.89 to 1.18$)$ & 0.004 & 0.712 \\
\hline Hallux valgus (presence) & 3.08 (0.23 to 41.79$)$ & 0.012 & 0.399 \\
\hline Lesser toe deformity (presence) & 2.72 (0.17 to 43.62$)$ & 0.014 & 0.480 \\
\hline \multicolumn{4}{|l|}{ Footwear fit ${ }^{e}$} \\
\hline Length (ill-fitting/lower median) ${ }^{f}$ & 0.23 (0.02 to 2.70$)$ & 0.061 & 0.240 \\
\hline Width (ill-fitting/lower median) ${ }^{f}$ & 0.89 (0.11 to 7.15$)$ & 0.042 & 0.915 \\
\hline
\end{tabular}

\footnotetext{
${ }^{\mathrm{a}}$ Adjusted for sex, age and body mass index.
}

${ }^{b}$ OxAFQ-C Footwear domain scores were dichotomised where the lowest quartile (item responses: always, very often) was classified as 'disability' and the upper three quartiles (item responses: never, rarely and sometimes) were classified as 'no disability'.

cOR: Odds ratio.

${ }^{\mathrm{d}}$ Arch Index multiplied by hundred.

e Logistic regression analyses performed for footwear fit were based on $n=48$ due to missing data.

fIIl-fitting: representing the greatest disparity between the participant's foot and footwear length and width dimensions; expressed as a percentage. The values that defined the lower median (ill-fitting) were as follows: length (shoe $14.3 \%$ shorter to $10.8 \%$ longer than foot), width (shoe $26.5 \%$ to $4.9 \%$ narrower than foot). 
reported scores [34]. Second, this is a cross-sectional study, so we cannot confirm the relationships identified in this study are causal. Third, we recruited participants from a Victorian (Australia) state cohort. Future research is required to confirm these findings in larger cohorts of children with DS to improve generalisability. Fourth, caution needs to be exercised when interpreting the study's findings in respect to analyses involving foot structure, as the measures of the severity of hallux valgus and presence of lesser toe deformity have not been validated in child and adolescent populations. Fifth, hallux valgus and inadequate footwear width were only responsible for between $10 \%$ and $14 \%$ of the variance in the OxAFQ-C domain scores; there are likely to be several other factors that were not investigated that may be associated with foot-specific disability in children with DS. In this regard, the OxAFQ-C was designed to assess disability associated with foot and/or ankle conditions, however our study only used foot conditions. Finally, lower limb muscle hypotonia and joint laxity have also been associated with muscle fatigue and gait abnormality in children with DS, similar to children with joint hypermobility syndrome [48-50]. Hence, inclusion of these variables in future analyses may lead to more predictive models.

\section{Conclusions}

Our study showed that foot posture and lesser toe deformities were not associated with foot-specific disability in children with DS. However, hallux valgus and the use of ill-fitting footwear, specifically footwear that was too narrow, were associated with increased levels of footspecific disability. These findings suggest that footwear education, and regular footwear assessments may be important for children and adolescents with DS, providing the associations observed in the current study prove to be causal. Further, findings from this study warrant the need for future studies to identify other factors that may be associated with foot-specific disability in children with DS. Ideally, future work should use prospective study designs, and be conducted across the lifespan of individuals with DS.

\section{Competing interests}

The authors declare that they have no competing interests.

\section{Authors' contributions}

All authors were involved with the study conception, design and participant recruitment. PQXL carried out data collection. PQXL and SEM conducted statistical analysis, interpreted data and drafted the manuscript. All authors were involved in critical revision and approval of the manuscript.

\section{Acknowledgements}

The authors would like to acknowledge the contributions of all the participants and their families. We would also like to acknowledge Down Syndrome Victoria (Australia) for advertising our study. The authors would also like to acknowledge Maria Auhl (Research assistant) and Jade Tan (Research fellow) for their assistance in data entry and staff of the Health Sciences Clinic at La Trobe University for the use of facilities. This study was funded by a grant received from the Lower Extremity and Gait Studies (LEGS) Program (La Trobe University, Melbourne, Victoria, Australia).

\section{Author details}

${ }^{1}$ Discipline of Podiatry, College of Science, Health and Engineering, La Trobe University, Bundoora, Victoria 3086, Australia. ${ }^{2}$ Discipline of Physiotherapy, College of Science, Health and Engineering, La Trobe University, Bundoora, Victoria 3086, Australia. ${ }^{3}$ Lower Extremity and Gait Studies Program, La Trobe University, Bundoora, Victoria 3086, Australia.

Received: 30 September 2014 Accepted: 31 January 2015

Published online: 12 February 2015

\section{References}

1. Elton TS, Sansom SE, Martin MM. Trisomy-21 gene dosage over-expression of miRNAs results in the haploinsufficiency of specific target proteins. RNA Biol. 2010;7(5):540-7.

2. Roizen NJ, Patterson D. Down's syndrome. Lancet. 2003;361(9365):1281-9.

3. Steingass KJ, Chicoine B, McGuire D, Roizen NJ. Developmental disabilities grown up: Down syndrome. J Dev Behav Pediatr. 2011;32(7):548-58.

4. Cowley PM, Ploutz-Snyder LL, Baynard T, Heffernan K, Jae SY, Hsu S, et al. Physical fitness predicts functional tasks in individuals with Down syndrome. Med Sci Sports Exerc. 2010;42(2):388-93.

5. Mercer VS, Lewis CL. Hip abductor and knee extensor muscle strength of children with and without Down syndrome. Pediatr Phys Ther. 2001;13(1):18-26.

6. Galli M, Cimolin V, Pau M, Costici P, Albertini G. Relationship between flat foot condition and gait pattern alterations in children with Down syndrome. J Intellect Disabil Res. 2014;58(3):269-76.

7. Rigoldi C, Galli M, Albertini G. Gait development during lifespan in subjects with Down syndrome. Res Dev Disabil. 2011;32(1):158-63.

8. Mik G, Gholve PA, Scher DM, Widmann RF, Green DW. Down syndrome: orthopedic issues. Curr Opin Pediatr. 2008;20(1):30-6.

9. Caird MS, Wills BP, Dormans JP. Down syndrome in children: the role of the orthopaedic surgeon. J Am Acad Orthop Surg. 2006;14(11):610-9.

10. Livingstone B, Hirst P. Orthopedic disorders in school children with Down's syndrome with special reference to the incidence of joint laxity. Clin Orthop Relat Res. 1986;207:74-6.

11. Diamond LS, Lynne D, Sigman B. Orthopedic disorders in patients with Down's syndrome. Orthop Clin North Am. 1981;12(1):57-71.

12. Pikora TJ, Bourke J, Bathgate K, Foley KR, Lennox N, Leonard H. Health conditions and their impact among adolescents and young adults with Down syndrome. PLoS One. 2014;9(5):e96868.

13. Mahan KT, Diamond E, Brown D. Podiatric profile of the Down's syndrome individual. J Am Podiatry Assoc. 1983;73(4):173-9.

14. Caselli MA, Cohen-Sobel E, Thompson J, Adler J, Gonzalez L. Biomechanical management of children and adolescents with Down syndrome. J Am Podiatr Med Assoc. 1991;81(3):119-27.

15. Merrick J, Ezra E, Josef B, Hendel D, Steinberg DM, Wientroub S. Musculoskeletal problems in down syndrome European paediatric orthopaedic society survey: the Israeli sample. J Pediatr Orthop B. 2000;9(3):185-92.

16. Concolino D, Pasquzzi A, Capalbo G, Sinopoli S, Strisciuglio P. Early detection of podiatric anomalies in children with Down syndrome. Acta Paediatr. 2006;95(1):17-20.

17. Prasher VP, Robinson L, Krishnan VH, Chung MC. Podiatric disorders among children with Down syndrome and learning disability. Dev Med Child Neurol. 1995;37(2):131-4.

18. Pau M, Galli M, Crivellini M, Albertini G. Foot-ground interaction during upright standing in children with Down syndrome. Res Dev Disabil. 2012;33(6):1881-7.

19. Cavanagh PR, Rodgers MM. The arch index: a useful measure from footprints. J Biomech. 1987;20(5):547-51.

20. Murley GS, Menz HB, Landorf KB. A protocol for classifying normal- and flat-arched foot posture for research studies using clinical and radiographic measurements. J Foot Ankle Res. 2009;2(1):22.

21. Gilmour JC, Burns $Y$. The measurement of the medial longitudinal arch in children. Foot Ankle Int. 2001;22(6):493-8. 
22. Igbigbi PS, Msamati BC. The footprint ratio as a predictor of pes planus: a study of indigenous Malawians. J Foot Ankle Surg. 2002;41(6):394-7.

23. Kanatli U, Yetkin H, Cila E. Footprint and radiographic analysis of the feet. J Pediatr Orthop. 2001;21(2):225-8.

24. Wearing SC, Hills AP, Byrne NM, Hennig EM, McDonald M. The arch index: a measure of flat or fat feet? Foot Ankle Int. 2004;25(8):575-81.

25. Mickle KJ, Steele JR, Munro BJ. The feet of overweight and obese young children: are they flat or fat? Obesity (Silver Spring). 2006;14(11):1949-53.

26. Murray J, Ryan-Krause P. Obesity in children with Down syndrome: Background and recommendations for management. Pediatr Nurs. 2010;36(6):314-9.

27. Rimmer JH, Yamaki K, Davis BM, Wang E, Vogel LC. Obesity and overweight prevalence among adolescents with disabilities. Prev Chronic Dis. 2011;8(2):A41.

28. Pau M, Galli M, Crivellini M, Albertini G. Relationship between obesity and plantar pressure distribution in youths with down syndrome. Am J Phys Med Rehabil. 2013;92(10):889-97.

29. Garrow AP, Papageorgiou A, Silman AJ, Thomas E, Jayson MIV, Macfarlane GJ. The grading of hallux valgus: The Manchester scale. J Am Podiatr Med Assoc. 2001;91(2):74-8.

30. Menz HB, Fotoohabadi MR, Wee E, Spink MJ. Validity of self-assessment of hallux valgus using the Manchester scale. BMC Musculoskelet Disord. 2010;11(1):1-6.

31. Menz HB, Munteanu SE. Radiographic validation of the Manchester scale for the classification of hallux valgus deformity. Rheumatology (Oxford). 2005;44(8):1061-6.

32. Menz HB, Lord SR. Foot pain impairs balance and functional ability in community-dwelling older people. J Am Podiatr Med Assoc. 2001;91(5):222-9.

33. Menz HB, Morris ME. Footwear characteristics and foot problems in older people. Gerontology. 2005;51(5):346-51.

34. Morris C, Doll HA, Wainwright A, Theologis T, Fitzpatrick R. The Oxford ankle foot questionnaire for children: scaling, reliability and validity. J Bone Joint Surg (Br). 2008;90(11):1451-6.

35. Morris C, Doll H, Davies N, Wainwright A, Theologis T, Willett K, et al. The Oxford Ankle Foot Questionnaire for children: responsiveness and longitudinal validity. Qual Life Res. 2009;18(10):1367-76.

36. Morris C, Doll H, Wainwright A, Davies N, Theologis T, Fitzpatrick R. The oxford ankle foot questionnaire for children: review of development and potential applications. Prosthet Orthot Int. 2010;34(3):238-44.

37. Faul F, Erdfelder E, Buchner A, Lang AG. Statistical power analyses using G*Power 3.1: tests for correlation and regression analyses. Behav Res Methods. 2009;41(4):1149-60.

38. Menz HB. Two feet, or one person? Problems associated with statistical analysis of paired data in foot and ankle medicine. Foot. 2004;14(1):2-5.

39. Tabachnick BG, Fidell LS. Multiple Regression. Chapter 5. In: Hartman S, editor. Using Multivariate Statistics. 5th ed. Boston: Pearson/Allyn \& Bacon; 2007. p. 117-94.

40. Jankowicz-Szymanska A, Mikolajczyk E, Wojtanowski W. The effect of the degree of disability on nutritional status and flat feet in adolescents with Down syndrome. Res Dev Disabil. 2013;34(11):3686-90.

41. Galli M, Cimolin V, Rigoldi C, Pau M, Costici P, Albertini G. The effects of low arched feet on foot rotation during gait in children with Down syndrome. J Intellect Disabil Res. 2014;58(8):758-64.

42. Evans AM, Rome K. A Cochrane review of the evidence for non-surgical interventions for flexible pediatric flat feet. Eur J Phys Rehabil Med. 2011;47(1):69-89.

43. Evans AM. Screening for foot problems in children: is this practice justifiable? J Foot Ankle Res. 2012;5(1):18.

44. Menz HB, Roddy E, Thomas E, Croft PR. Impact of hallux valgus severity on general and foot-specific health-related quality of life. Arthritis Care Res (Hoboken). 2011;63(3):396-404.

45. Nix SE, Vicenzino BT, Collins NJ, Smith MD. Gait parameters associated with hallux valgus: a systematic review. J Foot Ankle Res. 2013;6(1):9.

46. Chaiwanichsiri D, Tantisiriwat N, Janchai S. Proper shoe sizes for Thai elderly. Foot (Edinb). 2008;18(4):186-91.

47. Klein C, Groll-Knapp E, Kundi M, Kinz W. Increased hallux angle in children and its association with insufficient length of footwear: a community based cross-sectional study. BMC Musculoskelet Disord. 2009;10:159.

48. Rigoldi C, Galli M, Cimolin V, Camerota F, Celletti C, Tenore N, et al. Gait strategy in patients with Ehlers-Danlos syndrome hypermobility type and Down syndrome. Res Dev Disabil. 2012;33(5):1437-42.
49. Celletti C, Galli M, Cimolin V, Castori M, Albertini G, Camerota F. Relationship between fatigue and gait abnormality in joint hypermobility syndrome/Ehlers-Danlos syndrome hypermobility type. Res Dev Disabil. 2012;33(6):1914-8.

50. Galli M, Rigoldi C, Brunner R, Virji-Babul N, Giorgio A. Joint stiffness and gait pattern evaluation in children with Down syndrome. Gait Posture. 2008;28 (3):502-6.

\section{Submit your next manuscript to BioMed Central and take full advantage of:}

- Convenient online submission

- Thorough peer review

- No space constraints or color figure charges

- Immediate publication on acceptance

- Inclusion in PubMed, CAS, Scopus and Google Scholar

- Research which is freely available for redistribution 